\title{
Care of laryngectomy stomas in general practice
}

Christopher Jackson, Cameron Grigg, Megan Green, Roger Grigg

\section{Background}

Laryngectomy stomas are formed following excision of the larynx, usually for the treatment of an underlying malignancy. This is a permanent stoma in which the trachea is separated from the oesophagus and brought to an opening in the neck. The complication rate of laryngectomy stomas is reported to be more than $60 \%$.

\section{Objective}

The purpose of this article is to provide an overview of total laryngectomy stomas and inform general practitioners (GPs) of the frequently encountered complications, to improve stoma-related care and ultimately patient outcomes.

\section{Discussion}

Numerous early and late complications can be encountered by a GP, some of which are potentially life-threatening and require prompt recognition. Care of patients who have had a laryngectomy is multi-disciplinary, and GPs are often the first point of contact. The importance of a well-informed GP who can treat the patient and identify when they require referral to a stomal therapy nurse, speech pathologist or surgeon cannot be understated.
A LARYNGECTOMY STOMA is formed following total laryngectomy surgery for advanced or recurrent laryngeal cancer. It is a permanent stoma in which the trachea is brought to an opening in the anterior neck, as shown in Figure 1. Within Australia, the incidence of laryngeal cancer has increased from 553 patients diagnosed per year in 1982 to 606 in $2009 .{ }^{1}$ Although a proportion of these patients are treated with primary radiotherapy, the operative management rate for laryngeal cancer has also increased from 2.5 to 2.9 patients per 100,000 (2002 to 2012). ${ }^{1}$ While similar to abdominal stomas, which are more frequently encountered, there are many specific complications that can occur with laryngectomy stomas. There is a well-recognised lack of confidence among health professionals when managing laryngectomy stomas and their complications. ${ }^{2,3}$ The increased incidence of laryngeal cancer reinforces that, even though general practitioners (GPs) are part of a multi-disciplinary team in the care of patients who have had a laryngectomy, as primary health practitioners it is recommended that they are comfortable managing any initial complications that may present.

\section{Complications of laryngectomy stomas}

Complication rates of laryngectomy stomas are reported to be as high as $60 \% .^{4}$ Aside from a pharyngocutaneous fistula or stomal recurrence, which are early and late findings respectively, the variety of complications of laryngectomy stomas can occur at any stage throughout the post-operative period. Unlike abdominal stomas, many of these significant complications can therefore be late presentations, potentially after the patient has been discharged from surgical follow-up. In such situations, patients may see their GPs as their first point of contact.

\section{Early complications}

Bleeding

Bleeding from the stoma can be a common early presentation, and it should first be determined whether the patient is coughing up blood or simply identifying it at the stomal site. The patient and stoma should be examined closely (Figure 2) for the presence of granulation tissue at the skin edge or any wound breakdown if the operation was recent. Insensate granulation tissue can be treated with the application of silver nitrate cautery in the GP setting or a short course of topical antibiotic and corticosteroid ointment. ${ }^{5}$ Granulation tissue can also commonly occur around the voice prosthesis, which is inserted into the posterior wall of the trachea (Figure 3); if this occurs, then review by a speech pathologist is required so the prosthesis can be replaced.

It is important to quantify the amount of bleeding with the patient, as shown in Figure 2; less than $10 \mathrm{~mL}$ at once is not a concern. While extremely rare, tracheoinnominate fistulas have been described in patients post-laryngectomy, particularly if patients have a tracheostomy tube inserted. ${ }^{5}$ These can present initially with smaller sentinel bleeds, so if there 
is any concern, patients should be referred to the emergency department for review. ${ }^{6}$ Small-volume bleeding that the patient 'coughs' up can be due to vigorous suctioning but should prompt investigation for synchronous lung pathology, particularly given the patients' higher background risk.

\section{Pharyngocutaneous fistula}

Pharyngocutaneous fistula is the most common, and normally early, complication following total laryngectomy, with an incidence of up to $65 \%$ reported. ${ }^{7}$ It is an abnormal communication between the pharynx and cervical skin at the stoma or, more frequently, the surgical incision site. Examination will reveal a cutaneous defect, as shown in Figure 3, with salivary output also potentially seen. Treatment is normally conservative, and patients may present to their GPs for regular dressing changes, which are required to maintain integrity of the skin and prevent aspiration of saliva per stoma.

\section{Stomal stenosis}

Patients may present with concerns regarding reduced patency of the laryngectomy stoma. Stenosis of the post-laryngectomy stoma remains an ongoing concern, with a reported incidence in up to $22 \%$ of patients. ${ }^{8}$ It is typically associated with the administration of post-operative radiotherapy and the surgical technique initially used to form the stoma. ${ }^{8}$ It can be distressing for the patient, and they should be instructed to keep a laryngectomy tube inserted into the stoma to ensure patency until a speech pathologist and surgical review is arranged.

\section{Peristomal skin irritation}

Patients with a laryngectomy stoma can experience significant skin irritation and consequently peristomal breakdown, as similarly occurs with an abdominal stoma. As with abdominal stomas, a stomal therapy nurse (STN) is an expert in managing these conditions and should be contacted for assistance with dressing choice and alteration if necessary. Aside from a fistula, a common cause of irritation is the use of a heat and moisture exchange (HME) cover that is applied to the stoma to create a tight seal, preserving some of the moisture within the lower respiratory tract. The repeated removal and application of these appliances, or the glue used to secure the baseplate, can cause skin breakdown. An STN can assist with alteration of the appliance to one more tolerated. Leaving the stoma uncovered for at least two days is recommended for the situations above.

When reviewing the patient, an assessment for cellulitis is recommended, and treatment may commence if there is an underlying infective component. Patients presenting with significant erythema and desquamation of the anterior neck and stomal site during radiotherapy treatment will have radiation dermatitis. In this instance, the radiation oncology service completing the treatment can be notified, and they can direct treatment for the patient, which may consist of a short-course topical corticosteroid.
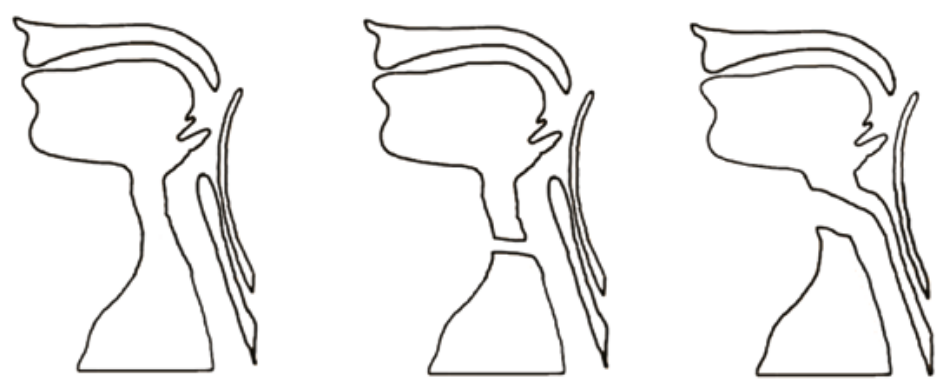

Figure 1. Schematic illustration of normal upper aerodigestive anatomy (left), tracheostomy (centre) and total laryngectomy (right)

\section{Stomal crusting}

Stomal crusting and mucous plugging are not only frustrating for the patient but can become life-threatening if acute airway obstruction develops. As shown in Figure 1, as a result of post-laryngectomy anatomy changes, patients lose the humidification effects of the upper airway, and inhaled air passes directly into the trachea. This can cause irritation to the trachea at the stomal site, leading to crusting and an over-production of mucous. ${ }^{9}$ If crusting and mucous plugging is a concern, the frequency of nebulised saline and tracheal suctioning should be increased or an HME considered, in consultation with a speech pathologist. If crusting is particularly bad at the stomal site itself, a laryngectomy tube coated in antibiotic and steroid ointment can be used and changed regularly.

\section{Acute airway obstruction}

If patients who have had a laryngectomy present with acute airway obstruction, assessment and management may be performed following the steps in Figure 4, with transfer to the emergency department urgently arranged. As is highlighted in Figure 4, the important difference in managing these patients is an emphasis on clearing any obstruction from the stoma if present, using either direct vision or a yankauer/soft suction catheter if available. If cardiopulmonary resuscitation is required, mouth to stoma breaths can be performed. If the patient requires ventilation, a paediatric face mask attached to a standard bag-valve-mask apparatus, if available, can be applied to the stoma.

\section{Late complications}

Voice prosthesis dislodgement/leakage Most patients who have had a laryngectomy will be regularly reviewed by a speech pathologist and otolaryngologist in the post-operative period. While a speech pathologist would normally be patients' first point of contact for any voice prosthesis complications, patients may present to their GPs out of hours. One of the more commonly encountered complications is leakage, either through or around 
the voice prosthesis. Leakage through the prosthesis can be addressed by brushing and flushing the prosthesis and then capping it if the leak persists. If leakage occurs around the prosthesis, follow-up with a speech pathologist is recommended. A common cause of leakage is chronic fungal overgrowth, so a trial of oral and systemic anti-fungals is also often useful. If the patient lives in a rural location and a speech pathologist is not available, the voice prosthesis should be removed and a catheter inserted; alternatively, a tracheostomy tube with a cuff can be inserted into the stoma to prevent leakage.
Dislodgement of the prosthesis can also occur. If the location of the prosthesis is unknown and/or the patient is experiencing dyspnoea, then it is assumed to be an airway foreign body and the patient should be referred to the emergency department urgently. In the absence of airway concerns, the priority

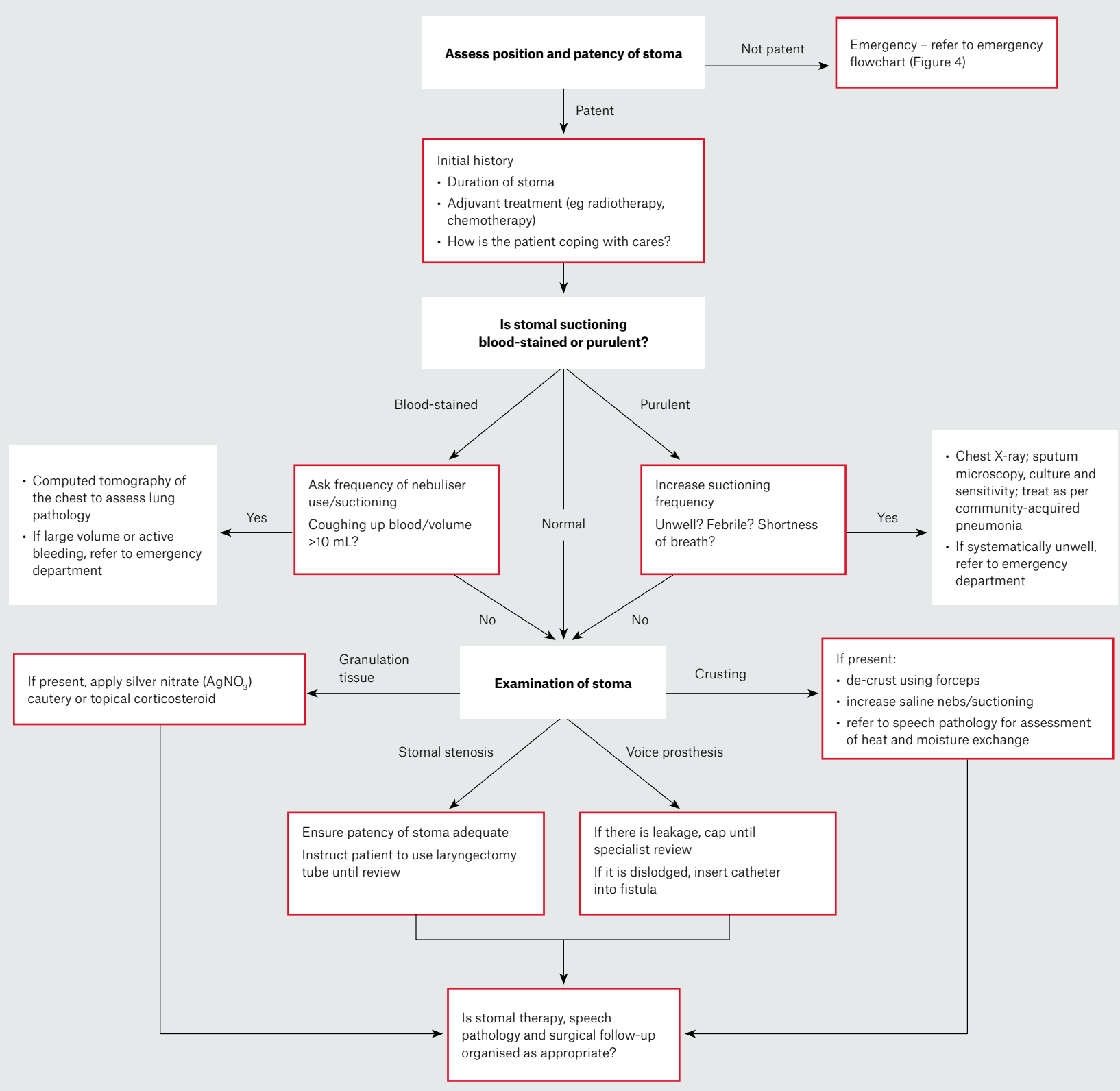

Figure 2. History and examination algorithm for patients who have had a laryngectomy 
is to maintain patency of the tracheooesophageal fistula, which can close within hours. ${ }^{10}$ All patients should have an appropriately sized catheter inserted into the fistula in this situation; if one is not available, a 14 Fr Foley's catheter with the balloon left uninflated can be used. A knot is tied at one end and the other end is inserted at least $6 \mathrm{~cm}$ into the fistula and down the oesophagus. Without insertion of the catheter, anything swallowed would be able to pass directly into the trachea. With a catheter in position, patients can follow up as required with a speech pathologist for replacement of the voice prosthesis.

\section{Stomal recurrence}

Stomal recurrence after laryngectomy is a complication feared by both patient and clinician and typically presents as an enlarging nodule or soft tissue mass at the stomal site within 1-2 years post-operatively. ${ }^{11}$ Unfortunately, stomal recurrence has an incidence of $3-15 \%$ in patients who have had a laryngectomy. ${ }^{11,12}$ If the treating GP is comfortable, a simple punch biopsy of the cutaneous aspect of the mass can be performed; however, it is recommended that these patients are referred for surgical review.

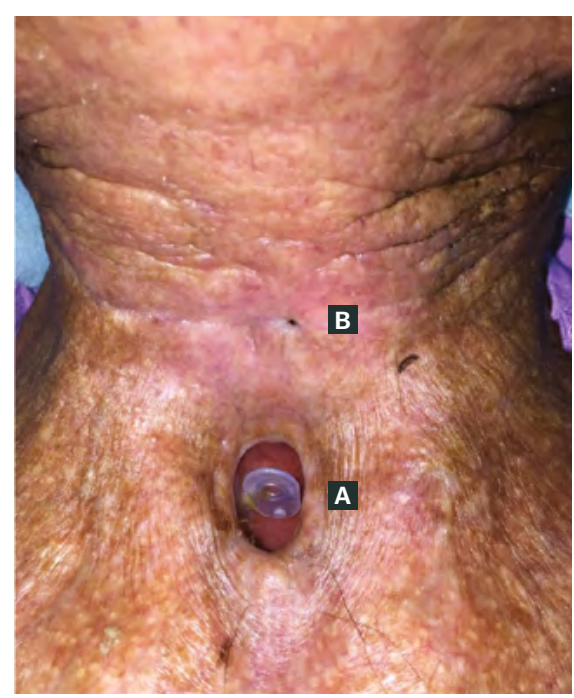

Figure 3. Laryngectomy stoma

A. Voice prosthesis appropriately positioned;

B. pharyngocutaneous fistula noted superiorly

\section{Psychological impact}

A total laryngectomy and the formation of a laryngectomy stoma is a significant life-changing operation for the patient that will permanently alter many aspects of their life. ${ }^{13}$ Patients are confronted with the effects of the operation every day, which include the loss of sense of smell and taste, difficulty passing bowel motions and, most importantly, the way they communicate within society. These functional and psychological changes occur at a time when the patient is not only recovering from an extensive operation but has also been diagnosed with an advanced malignancy. While holistic care is provided within the hospital, on discharge patients may present to GPs with not only stomal complications, but also those of a significant psychological nature. Despite these changes, research shows that, with properly coordinated multi-disciplinary care, most patients who have had a laryngectomy judge their post-op quality of life to be above average. ${ }^{14}$

Aside from swimming and contact sports, patients can resume normal activities. While it is safe to resume sexual activity, many patients will struggle with a lack of self-confidence regarding the

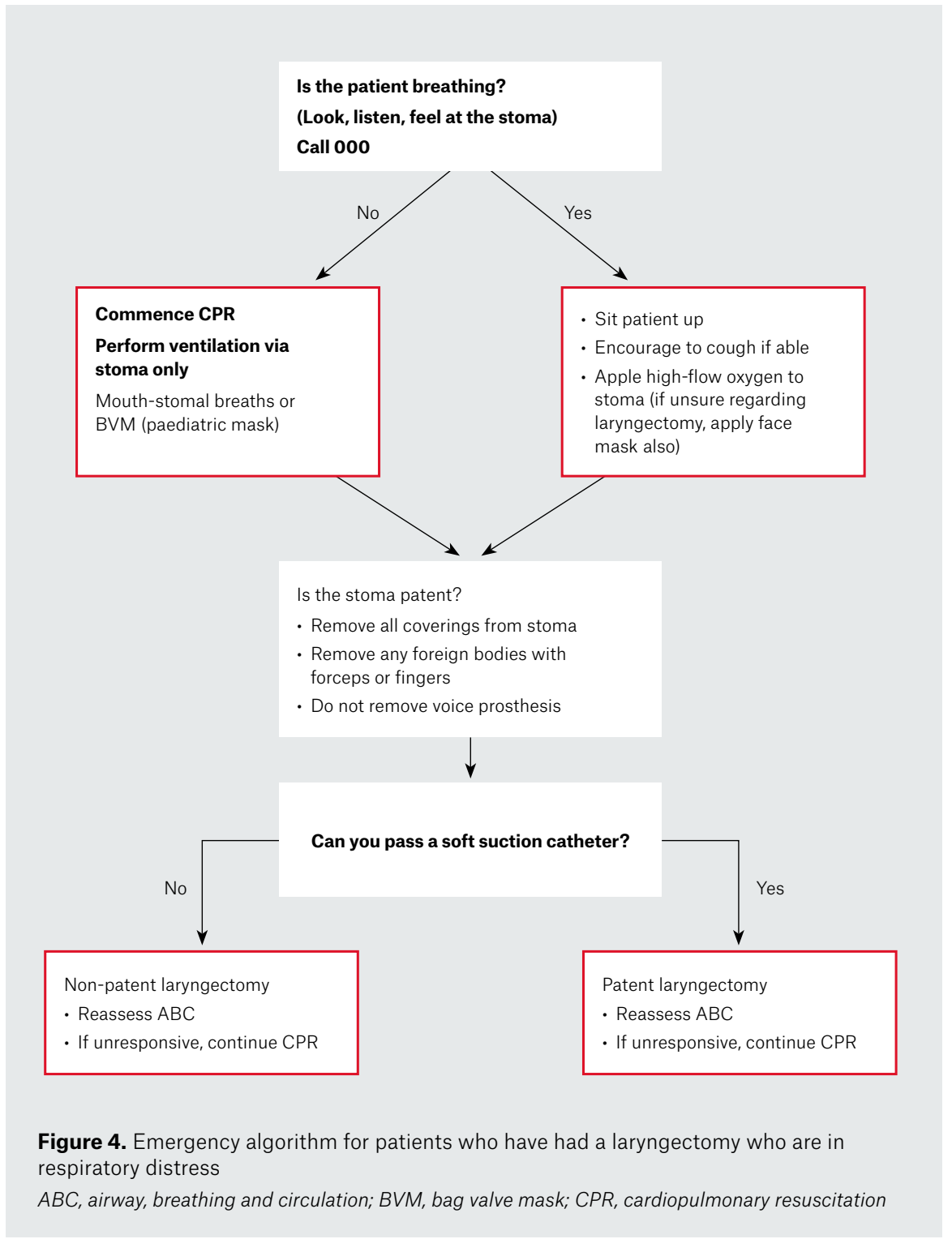


stoma, which can be addressed with a simple scarf or neck tie. ${ }^{15}$ The adjustment of the patient's partner, who is often the primary carer assisting with the stoma, should also be explored particularly for the presence of carer fatigue. Loss of control and frustration that communication is always 'over their head' is the most commonly reported concern by partners of patients who have had a laryngectomy. ${ }^{16}$ Therefore, the ability to have concerns addressed by a familiar face such as their regular GP is significant for both patient and partner. Ensuring patients and their carers are aware of the 'Head and neck cancer' or 'Laryngectomee' support groups available online, or within their local area, can prove to be a simple but significant factor in their progress to recovery.

\section{Conclusion}

The importance of correctly managing simple laryngectomy stomal complications in the primary care setting cannot be understated. As the first point of contact, GPs have an important role within the multi-disciplinary team and may need to refer patients to an STN, speech pathologist or surgeon as indicated. There is little doubt that the correct functioning of this multi-disciplinary team plays a significant role not only in the positive functional, but also psychological, outcome of patients. ${ }^{14}$

\section{Authors}

Christopher Jackson BSc, MBBS, Principal House Officer, Department of Otolaryngology and Head and Neck Surgery, Toowoomba Hospital, Qld. christopher.jackson3@health.qld.gov.au

Cameron Grigg BSc, MBBS, Principal House Officer, Department of Otolaryngology and Head and Neck Surgery, Toowoomba Hospital, Qld

Megan Green BNsg, GradCert Wound \& Stomal Therapy, Clinical Nurse Consultant, Department of Otolaryngology and Head and Neck Surgery, Toowoomba Hospital, Qld

Roger Grigg BSc, MBBS, RACS, Associate Professor, University of Queensland, Qld; Director of Ear, Nose and Throat Surgery, Department of Surgery, Toowoomba Hospital, Qld

Competing interests: None.

Funding: None.

Provenance and peer review: Not commissioned, externally peer reviewed.

\section{References}

1. Australian Institute of Health and Welfare. Head and neck cancers in Australia. Canberra: AlHW, 2014. Available at www.aihw.gov.au/getmedia/ bdccebf2-dbe6-44e2-9104-8461d7e7c165/16933. pdf.aspx?inline=true [Accessed 27 March 2019].
2. Killick N, Cameron C, Kulbacki H, Malik V, Kumar N. Development of a tracheostomy/ laryngectomy care workshop for ward staff in non-ENT wards. Int J Surg 2014;12(3):83. doi: 10.1016/j.ijsu.2014.08.157.

3. Lau K, Crouch C. Tracheostomies and laryngectomies: Are junior doctors confident in managing these in an emergency? Int J Surg 2014;12(3):72. doi: 10.1016/j.ijsu.2014.08.103.

4. Hanansano MM, Lin D, Wax MK, Rosenthal EL. Closure of laryngectomy defects in the age of chemoradiation therapy. Head Neck 2012;34(4):580-88. doi: 10.1002/hed.21712

5. Sobol SE, Keswani S, Parvadia JK, Crombleholme T, Potsic WP. Effect of corticosteroid-antibiotic agents on granulation tissue in a murine model. Arch Otolaryngol Head Neck Surg 2005;131(4):330-35. doi: 10.1001/ archotol.131.4.330.

6. Sengor GA, Aydin O. Tracheo-innominate artery fistula in a laryngectomized patient. Kulak Burun Bogaz Ithis Derg 2008;18(2):106-09.

7. Dedivitis RA, Ribeiro KCB, Castro MAF, Nascimento PC. Pharyngocutaneous fistula following total laryngectomy. Acta Otorhinolaryngol Ital 2007;27(1):2-5.

8. Griffith GR, Luce EA. Tracheal stomal stenosis after laryngectomy. Plast Reconstr Surg 1982;70(6):694-98. doi: 10.1097/00006534198212000-00006.

9. Patel A, Theokli C. Nebulised N-acetylcysteine used in acute tracheostoma obstruction. J Laryngol Otol 2014;128(12):1123-24. doi: 10.1017/ S0022215114002175.

10. St George's University Hospitals. Caring for a stoma and voice prosthesis after a total laryngectomy. London: NHS Foundation Trust, 2018. Available at www.stgeorges.nhs.uk/ wp-content/uploads/2018/03/ENT_SAVCD_01. pdf [Accessed 27 March 2019].

11. University of lowa Health Care. Treatment of stomal recurrence. lowa: University of lowa Healthcare, 2017. Available at https://medicine. uiowa.edu/iowaprotocols/treatment-stomalrecurrence [Accessed 27 March 2019].

12. Zhao H, Ren J, Zhuo X, Ye H, Zou J, Liu S. Stomal recurrence after total laryngectomy: A clinicopathological multivariate analysis. Am J Clin Oncol 2009;32(2):154-57. doi: 10.1097/ COC.0b013e31817e6ee3.

13. Noonan BJ, Hegarty J. The impact of total laryngectomy: The patient's perspective. Oncol Nurs Forum 2010;37(3):293-301. doi: 10.1188/10. ONF.293-301.

14. Braz DSA, Ribas MM, Dedivitis RA, Nishimoto IN, Barros AP. Quality of life and depression in patients undergoing total and partial laryngectomy. Clinics 2005;60(2):135-42. doi: 10.1590/S1807-59322005000200010.

15. Monga U, Tan G, Ostermann HJ, Monga TN. Sexuality in head and neck cancer patients. Arch Phys Med Rehabil 1997;78(3):298-304. doi: 10.1016/S0003-9993(97)90038-1.

16. Offerman MPJ, Pruyn JFA, de Boer MF, Busschbach JJV, Baatenburg de Jong RJ. Psychosocial consequences for partners of patients after total laryngectomy and for the relationship between patients and partners. Oral Oncology 2015;51(4):389-98. doi: 10.1016/j. oraloncology.2014.12.008 